\title{
@creative
commons
}

ISSN 1855-3966 (printed edn.), ISSN 1855-3974 (electronic edn.)

\author{
ARS MATHEMATICA CONTEMPORANEA 20 (2021) 199-208 \\ https://doi.org/10.26493/1855-3974.2043.fbb \\ (Also available at http://amc-journal.eu)
}

\section{The average genus for bouquets of circles and dipoles}

\author{
Jinlian Zhang \\ School of Mathematics and Statistics, Hunan University of Finance and Economics, \\ Changsha, P. R. China \\ Xuhui Peng * \\ MOE-LCSM, School of Mathematics and Statistics, Hunan Normal University, \\ Changsha, P. R. China \\ Yichao Chen ${ }^{\dagger}$ \\ Department of Mathematics and Physics, Suzhou University of Science and Technology, \\ Suzhou, P. R. China
}

Received 9 July 2019, accepted 6 February 2021, published online 29 October 2021

\begin{abstract}
The bouquet of circles $B_{n}$ and dipole graph $D_{n}$ are two important classes of graphs in topological graph theory. For $n \geq 1$, we give an explicit formula for the average genus $\gamma_{\text {avg }}\left(B_{n}\right)$ of $B_{n}$. By this expression, one easily sees $\gamma_{\text {avg }}\left(B_{n}\right)=\frac{n-\ln n-\gamma+1-\ln 2}{2}+o(1)$, where $\gamma$ is the Euler-Mascheroni constant. Similar results are obtained for $D_{n}$. Our method mainly depends on the technique of generating series and the knowledge in ordinary differential equations.
\end{abstract}

Keywords: Average genus, bouquet of circles, dipole, ordinary differential equation.

Math. Subj. Class. (2020): 05C10

\footnotetext{
*Corresponding author. Xuhui Peng was supported by NNSFC (No. 12071123), the Scientific Research Project of Hunan Province Education Department (No. 20A329) and the Construct Program of the Key Discipline in Hunan Province.

† Yichao Chen was supported by NNSFC under Grant No. 11471106.

E-mail addresses: jinlian916@hnu.edu.cn (Jinlian Zhang), xhpeng@hunnu.edu.cn (Xuhui Peng), ycchen@hnu.edu.cn (Yichao Chen)
} 


\section{Introduction and main results}

A graph $G=(V(G), E(G))$ is permitted to have both loops and multiple edges. An embedding of a graph $G$ into an orientable surface $O_{k}$ is a cellular embedding, i.e., the interior of every face is homeomorphic to an open disc. The subscript in $O_{k}$ is the genus of the orientable surface $O_{k}$, for $k \geq 0$. We denote the number of cellular embeddings of $G$ on the surface $O_{k}$ by $g_{k}(G)$, where, by the number of embeddings, we mean the number of equivalence classes under ambient isotopy. The genus polynomial of a graph $G$ is given by

$$
\Gamma_{G}(x)=\sum_{k \geq 0} g_{k}(G) x^{k}
$$

This sequence $\left\{g_{k}(G), k=0,1,2, \ldots\right\}$ is called the genus distribution of the graph $G$. For a graph $G$, it is well known that the total number of cellular embeddings is $\prod_{v \in V(G)}\left(d_{G}(v)-1\right)$ !, where $d_{G}(v)$ is the degree of the vertex $v$ in $G$. For example, see [13, Chapter 3]. Hence,

$$
\Gamma_{G}(1)=\sum_{k \geq 0} g_{k}(G)=\prod_{v \in V(G)}\left(d_{G}(v)-1\right) !
$$

The average genus $\gamma_{\mathrm{avg}}(G)$ of the graph $G$ is the expected value of the genus random variable, over all labeled 2-cell orientable embeddings of $G$, using the uniform distribution. In other words, the average genus of $G$ is

$$
\gamma_{\mathrm{avg}}(G)=\frac{\Gamma_{G}^{\prime}(1)}{\Gamma_{G}(1)}=\sum_{k=0}^{\infty} k \cdot \frac{g_{k}(G)}{\Gamma_{G}(1)} .
$$

The study of the average genus of a graph began by Gross and Furst [9], and was much further developed by Chen and Gross [1, 2, 3]. Two lower bounds were obtained in [4] for the average genus of two kinds of graphs. In [19], Stahl gave the asymptotic result for the average genus of linear graph families. The exact values for the average genus of smallorder complete graphs, closed-end ladders, and cobblestone paths were derived by White [22]. More references are the following: [5, 10, 15, 17, 20] etc. For a general background in topological graph theory, we refer the reader to see Gross and Tucker [13] or White [21].

One of the purposes of the paper is to give an explicit expression of the average genus for a bouquet of circles. By a bouquet of circles, or more briefly, a bouquet, we mean a graph with one vertex and some self-loops. In particular, the bouquet with $n$ self-loops is denoted by $B_{n}$. Figure 1 shows the graphs $B_{1}, B_{2}, B_{3}$. The bouquets $\left\{B_{n}, n \geq 1\right\}$ are very important graphs in topological graph theory. First, since any connected graph can be reduced to a bouquet by contracting a spanning tree to a point, bouquets are fundamental building blocks of topological graph theory. Second, as shown in [8, 12], Cayley graphs and many other regular graphs are covering spaces of bouquets.

For the genus distribution of $B_{n}$, Gross, Robbins and Tucker [11] proved that the numbers $g_{k}\left(B_{n}\right)$ of embeddings of the $B_{n}$ in an oriented surface of genus $k$ satisfy the following recurrence for $n>2$,

$$
\begin{aligned}
(n+1) g_{k}\left(B_{n}\right)=4(2 n-1)(2 n-3)(n-1)^{2}(n-2) g_{k-1}\left(B_{n-2}\right) & \\
& +4(2 n-1)(n-1) g_{k}\left(B_{n-1}\right)
\end{aligned}
$$




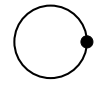

$B_{1}$

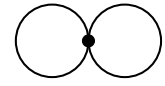

$B_{2}$

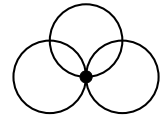

$B_{3}$

Figure 1: The bouquets $B_{1}, B_{2}$, and $B_{3}$.

with initial conditions

$$
\begin{aligned}
& g_{k}\left(B_{0}\right)=1 \text { for } k=0 \text { and } g_{k}\left(B_{0}\right)=1 \text { for } k>0, \\
& g_{k}\left(B_{1}\right)=1 \text { for } k=0 \text { and } g_{k}\left(B_{1}\right)=1 \text { for } k>0 .
\end{aligned}
$$

With the aid of an edge-attaching surgery technique, the total embedding polynomial of $B_{n}$ was computed in [14]. Stahl [18] also did some research on the average genus of $B_{n}$. By [18, Theorem 2.5] and the definition of Euler-Mascheroni constant, one easily sees that

$$
\lim _{n \rightarrow \infty}\left(\gamma_{\text {avg }}\left(B_{n}\right)-\left(\frac{n+1}{2}-\frac{1}{2} \sum_{k=1}^{2 n} \frac{1}{k}\right)\right)=0 .
$$

To achieve this, Stahl made many accurate estimates on the unsigned Stirling numbers $s(n, k)$ of the first kind. In this paper, using knowledge in ordinary differential equations and Taylor's formula, we derive an explicit expression of $\gamma_{\mathrm{avg}}\left(B_{n}\right)$. By this expression, (1.4) follows immediately. Our methods are totally different from that in [18] and we do not need to make estimates on $s(n, k)$. In Section 2, we will give the computation of $\gamma_{\text {avg }}\left(B_{n}\right)$ in detail.

A dipole with $n$ edges, denoted by $D_{n}$, has two vertices joined by $n$ edges. Figure 2 shows the graphs $D_{1}, D_{2}, D_{3}$.

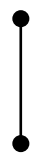

$D_{1}$

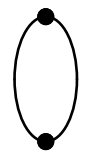

$D_{2}$

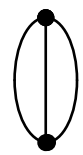

$D_{3}$

Figure 2: The dipoles $D_{1}, D_{2}$, and $D_{3}$.

Another purpose of this paper is to give an explicit expression of the average genus for dipole $D_{n}$. The dipole, like the bouquet, is useful as a voltage graph. See [21] for example. Moreover, hypermaps correspond with the 2-cell embeddings of the dipole. The genus distribution of $D_{n}$ is given by [14] and [16].

In Lemma 2.1 below, we obtain the following recurrence relation for $\gamma_{\text {avg }}\left(B_{n}\right)$

$$
(n+1) \gamma_{\mathrm{avg}}\left(B_{n}\right)=2 \gamma_{\mathrm{avg}}\left(B_{n-1}\right)+(n-1)\left(\gamma_{\mathrm{avg}}\left(B_{n-2}\right)+1\right) .
$$

The most popular way to deal with sequences of numbers is to manipulate infinite series that "generate" those sequences. For instance, see [6, 7]. We apply this method to 
the calculation of $\gamma_{\text {avg }}\left(B_{n}\right)$. Multiplying both sides of (1.5) by $t^{n}$ and summing on $n \geq$ 1 , the generating function $u(t)=\sum_{n>1} \gamma_{\text {avg }}\left(B_{n}\right) t^{n}$ will satisfy an ordinary differential equation. We solve this differential equation with the aid of a computer system and find an explicit expression for $\gamma_{\text {avg }}\left(B_{n}\right)$ by expanding $u(t)$ as a power series in $t$. The calculation of $\gamma_{\text {avg }}\left(D_{n}\right)$ is similar to that in $\gamma_{\text {avg }}\left(B_{n}\right)$. But the processes are more complicated, so we still give their details in Section 3.

\section{The average genus of $B_{n}$}

We begin by proving the following lemma.

Lemma 2.1. The following recurrence relation holds for the average genus $\gamma_{\mathrm{avg}}\left(B_{n}\right)$ of $B_{n}$

$$
(n+1) \gamma_{\mathrm{avg}}\left(B_{n}\right)=2 \gamma_{\mathrm{avg}}\left(B_{n-1}\right)+(n-1)\left(\gamma_{\mathrm{avg}}\left(B_{n-2}\right)+1\right)
$$

with initial conditions $\gamma_{\text {avg }}\left(B_{1}\right)=0, \gamma_{\text {avg }}\left(B_{2}\right)=\frac{1}{3}$.

Proof. Multiplying both sides of (1.2) by $x^{k}$ and summing on $k \geq 0$, it holds that

$$
\begin{array}{rl}
\sum_{k \geq 0}(n+1) g_{k}\left(B_{n}\right) x^{k}=\sum_{k \geq 0} & 4(2 n-1)(2 n-3)(n-1)^{2}(n-2) g_{k-1}\left(B_{n-2}\right) x^{k} \\
& +\sum_{k \geq 0} 4(2 n-1)(n-1) g_{k}\left(B_{n-1}\right) x^{k}
\end{array}
$$

Hence, the genus polynomial $\Gamma_{B_{n}}(x)$ satisfies the following recurrence

$$
\begin{aligned}
(n+1) \Gamma_{B_{n}}(x)=4(2 n-1)(2 n-3)(n-1)^{2}(n-2) & \cdot x \cdot \Gamma_{B_{n-2}}(x) \\
& +4(2 n-1)(n-1) \Gamma_{B_{n-1}}(x) .
\end{aligned}
$$

Differentiating both sides of (2.3) and taking $x=1$ lead to

$$
\begin{aligned}
& (n+1) \Gamma_{B_{n}}^{\prime}(1)=4(2 n-1)(2 n-3)(n-1)^{2}(n-2) \cdot \Gamma_{B_{n-2}}^{\prime}(1) \\
& \quad+4(2 n-1)(2 n-3)(n-1)^{2}(n-2) \cdot \Gamma_{B_{n-2}}(1)+4(2 n-1)(n-1) \Gamma_{B_{n-1}}^{\prime}(1) .
\end{aligned}
$$

Applying (1.1) to the graph $B_{n}$ yields $\Gamma_{B_{n}}(1)=(2 n-1)$ !. Dividing both sides of the above equality by $\Gamma_{B_{n}}(1)$, by the definition of average genus, one arrives at

$$
(n+1) \gamma_{\mathrm{avg}}\left(B_{n}\right)=2 \gamma_{\mathrm{avg}}\left(B_{n-1}\right)+(n-1)\left(\gamma_{\mathrm{avg}}\left(B_{n-2}\right)+1\right) .
$$
pleted.

A direct calculation gives rise to $\gamma_{\mathrm{avg}}\left(B_{1}\right)=0$ and $\gamma_{\mathrm{avg}}\left(B_{2}\right)=\frac{1}{3}$. The proof is com-

The main purpose of this section is to prove the following theorem.

Theorem 2.2. The average genus of $B_{n}$ is given by

$$
\gamma_{\text {avg }}\left(B_{n}\right)=\frac{n+1}{2}-\sum_{m=0}^{n-1} \frac{1+(-1)^{m}}{2(m+1)}-\frac{1+(-1)^{n}}{4(n+1)} .
$$

In particular, we have

$$
\gamma_{\text {avg }}\left(B_{n}\right)=\frac{n-\ln n-\gamma+1-\ln 2}{2}+o(1),
$$

where $\gamma \approx 0.5772$ is the Euler-Mascheroni constant. 
Proof. For $n \leq 0$, we define $\gamma_{\text {avg }}\left(B_{n}\right)=0$ so that (2.1) holds for any integer $n \geq 1$. For the simplicity of writing, we use $a_{n}$ to denote $\gamma_{\text {avg }}\left(B_{n}\right)$ in the proof. Multiplying both sides of (2.1) by $t^{n}$ and summing on $n \geq 1$, we obtain

$$
\sum_{n \geq 1}(n+1) a_{n} t^{n}=2 \sum_{n \geq 1} a_{n-1} t^{n}+\sum_{n \geq 1}(n-1)\left(a_{n-2}+1\right) t^{n} .
$$

Let $u(t)=\sum_{n \geq 1} a_{n} t^{n}$. Then, with the help of (2.5), we obtain

$$
\begin{aligned}
\left(t \cdot \sum_{n \geq 1} a_{n} t^{n}\right)^{\prime} & =2 t \cdot \sum_{n \geq 1} a_{n-1} t^{n-1}+\sum_{n \geq 1}(n-2) a_{n-2} t^{n}+\sum_{n \geq 1} a_{n-2} t^{n}+\sum_{n \geq 1}(n-1) t^{n} \\
& =2 t u(t)+t^{3} \sum_{n \geq 1}(n-2) a_{n-2} t^{n-3}+t^{2} u(t)+t^{2} \cdot\left(\sum_{n \geq 2} t^{n-1}\right)^{\prime},
\end{aligned}
$$

that is

$$
\begin{aligned}
(t u(t))^{\prime} & =2 t u(t)+t^{3} \sum_{n \geq 3}(n-2) a_{n-2} t^{n-3}+t^{2} u(t)+t^{2}\left(\frac{t}{1-t}\right)^{\prime} \\
& =2 t u(t)+t^{3} \sum_{n \geq 1} n a_{n} t^{n-1}+t^{2} u(t)+t^{2}\left(\frac{t}{1-t}\right)^{\prime} \\
& =2 t u(t)+t^{3} u^{\prime}(t)+t^{2} u(t)+t^{2}\left(\frac{t}{1-t}\right)^{\prime}
\end{aligned}
$$

which implies that $u(t)$ satisfies the following equation

$$
\left(t-t^{3}\right) u^{\prime}(t)+\left(1-2 t-t^{2}\right) u(t)=\frac{t^{2}}{(1-t)^{2}}
$$

with initial condition $u(0)=0$. Since the above equation is a first order linear differential equation, we can solve it directly and obtain its solution:

$$
u(t)=\frac{-\left(t^{2}-1\right) \ln (1-t)+\left(t^{2}-1\right) \ln (t+1)+2 t}{4(t-1)^{2} t} .
$$

Denote

$$
u_{1}(t)=\frac{1}{2(t-1)^{2}}, \quad u_{2}(t)=-\frac{(t+1) \ln (1-t)}{4(t-1) t}, \quad u_{3}(t)=\frac{(t+1) \ln (t+1)}{4(t-1) t} .
$$

Then, clearly, $u(t)=u_{1}(t)+u_{2}(t)+u_{3}(t)$. Using Taylor's formula, we get

$$
u_{1}(t)=\sum_{n \geq 0} \frac{n+1}{2} t^{n}
$$

and

$$
u_{2}(t)=\frac{1}{4}(1+t) \cdot \frac{1}{1-t} \cdot \frac{\ln (1-t)}{t}=\frac{1}{4}(1+t) \cdot \sum_{\ell \geq 0} t^{\ell} \cdot \sum_{m \geq 0}\left(-\frac{1}{m+1} t^{m}\right)
$$




$$
=\frac{1}{4}(1+t) \cdot \sum_{n \geq 0} \sum_{m=0}^{n}\left(-\frac{1}{m+1}\right) t^{n}=\sum_{n \geq 0} b_{n} t^{n},
$$

where $b_{0}=-\frac{1}{4}$ and $b_{n}=\frac{1}{4}\left[\sum_{m=0}^{n}\left(-\frac{1}{m+1}\right)+\sum_{m=0}^{n-1}\left(-\frac{1}{m+1}\right)\right], n \geq 1$. Also by the Taylor's formula,

$$
\begin{aligned}
u_{3}(t) & =-\frac{1}{4}(1+t) \cdot \frac{1}{1-t} \cdot \frac{\ln (1+t)}{t}=-\frac{1}{4}(1+t) \cdot \sum_{\ell \geq 0} t^{\ell} \cdot \sum_{m \geq 0} \frac{(-1)^{m}}{m+1} t^{m} \\
& =-\frac{1}{4}(1+t) \cdot \sum_{n \geq 0} \sum_{m=0}^{n} \frac{(-1)^{m}}{m+1} t^{n}=\sum_{n \geq 0} c_{n} t^{n},
\end{aligned}
$$

where $c_{0}=-\frac{1}{4}$ and

$$
c_{n}=-\frac{1}{4}\left[\sum_{m=0}^{n} \frac{(-1)^{m}}{m+1}+\sum_{m=0}^{n-1} \frac{(-1)^{m}}{m+1}\right], \quad n \geq 1 .
$$

It follows from $(2.7)-(2.9)$ that

$$
\begin{aligned}
a_{n}=\frac{n+1}{2}+b_{n}+c_{n}=\frac{n+1}{2} & +\frac{1}{4}\left[\sum_{m=0}^{n}\left(-\frac{1}{m+1}\right)+\sum_{m=0}^{n-1}\left(-\frac{1}{m+1}\right)\right] \\
& -\frac{1}{4}\left[\sum_{m=0}^{n} \frac{(-1)^{m}}{m+1}+\sum_{m=0}^{n-1} \frac{(-1)^{m}}{m+1}\right]
\end{aligned}
$$

which yields (2.4). In view of

$$
\gamma=\lim _{n \rightarrow+\infty}\left[\sum_{m=0}^{n} \frac{1}{m+1}-\ln n\right] \text { and } \lim _{n \rightarrow+\infty} \sum_{m=0}^{n-1} \frac{(-1)^{m}}{m+1}=\ln 2,
$$

we complete the proof of (2.2).

\section{The average genus of $D_{n}$}

Our first purpose is to show the following lemma.

Lemma 3.1. The following recurrence relation holds for the average genus $\gamma_{\mathrm{avg}}\left(D_{n}\right)$ of $D_{n}$

$$
n(n+2) \gamma_{\mathrm{avg}}\left(D_{n+1}\right)=(2 n+1) \gamma_{\mathrm{avg}}\left(D_{n}\right)+\left(n^{2}-1\right) \cdot \gamma_{\mathrm{avg}}\left(D_{n-1}\right)+n^{2}
$$

with initial conditions $\gamma_{\text {avg }}\left(D_{1}\right)=\gamma_{\text {avg }}\left(D_{2}\right)=0$.

Proof. By [16, Theorem 5.2], we obtain

$(n+2) g_{k}\left(D_{n+1}\right)=n(2 n+1) g_{k}\left(D_{n}\right)+n^{3}(n-1)^{2} g_{k-1}\left(D_{n-1}\right)-n(n-1)^{2} g_{k}\left(D_{n-1}\right)$.

Applying (1.1) to the graph $D_{n+1}$ yields $\Gamma_{D_{n+1}}(1)=(n !)^{2}$. Following the lines in the proof of Lemma 2.1, we derive the recurrence relation (3.1).

The initial conditions $\gamma_{\text {avg }}\left(D_{1}\right)=\gamma_{\text {avg }}\left(D_{2}\right)=0$ are due to a direct calculation. The proof is finished. 
The main purpose of this section is to prove the following theorem.

Theorem 3.2. $\gamma_{\text {avg }}\left(D_{1}\right)=\gamma_{\text {avg }}\left(D_{2}\right)=0$ and for $n \geq 3$, we have

$$
\begin{aligned}
\gamma_{\mathrm{avg}}\left(D_{n}\right)=n\left[\frac{1}{2} \sum_{m=4}^{n+1} \frac{(-1)^{m}\left(4 m^{2}-12 m+6\right)}{(m-3)(m-2)(m-1) m}+\frac{1}{6}\right]-\frac{1}{2} \sum_{m=1}^{n+1} \frac{1}{m} \\
-\sum_{m=4}^{n+1} \frac{(-1)^{m}\left(2 m^{2}-6 m+3\right)}{(m-3)(m-1) m}+\frac{7}{12} .
\end{aligned}
$$

In particular, we have

$$
\gamma_{\text {avg }}\left(D_{n}\right)=\frac{n-\ln n-\gamma}{2}+o(1)
$$

where $\gamma \approx 0.5772$ is the Euler-Mascheroni constant.

Proof. First, we give a proof of (3.2). For the simplicity of writing, we use $a_{n}$ to denote $\gamma_{\text {avg }}\left(D_{n}\right)$ in the proof. Let $u(t)=\sum_{n \geq 1} a_{n} t^{n-3}=\sum_{n \geq 2} a_{n+1} t^{n-2}$. Multiplying both sides of (3.1) by $t^{n-2}$ and summing on $n \geq 2$, we obtain

$$
\begin{aligned}
\sum_{n \geq 2} n(n+2) a_{n+1} t^{n-2}=\sum_{n \geq 2}(2 n & +1) a_{n} t^{n-2} \\
& +\sum_{n \geq 2}\left(n^{2}-1\right) a_{n-1} t^{n-2}+\sum_{n \geq 2} n^{2} t^{n-2} .
\end{aligned}
$$

Since

$$
\begin{aligned}
u^{\prime}(t) & =\sum_{n \geq 2}(n-2) a_{n+1} t^{n-3} \\
u^{\prime \prime}(t) & =\sum_{n \geq 2}(n-2)(n-3) a_{n+1} t^{n-4}
\end{aligned}
$$

it follows that

$$
\begin{aligned}
\sum_{n \geq 2} n(n+2) a_{n+1} t^{n-2} & =\sum_{n \geq 2}[(n-2)(n-3)+7(n-2)+8] a_{n+1} t^{n-2} \\
& =t^{2} u^{\prime \prime}(t)+7 t u^{\prime}(t)+8 u(t), \\
\sum_{n \geq 2}(2 n+1) a_{n} t^{n-2} & =\sum_{n \geq 2}(2 n+3) a_{n+1} t^{n-1}=\sum_{n \geq 2}(2(n-2)+7) a_{n+1} t^{n-1} \\
& =2 t^{2} u^{\prime}(t)+7 t u(t), \\
\sum_{n \geq 2}\left(n^{2}-1\right) a_{n-1} t^{n-2} & =\sum_{n \geq 4}\left(n^{2}-1\right) a_{n-1} t^{n-2}=\sum_{n \geq 2}\left(n^{2}+4 n+3\right) a_{n+1} t^{n} \\
& =\sum_{n \geq 2}[(n-2)(n-3)+9(n-2)+15] a_{n+1} t^{n} \\
& =t^{4} u^{\prime \prime}(t)+9 t^{3} u^{\prime}(t)+15 t^{2} u(t), \\
\sum_{n \geq 2} n^{2} t^{n-2} & =\sum_{n \geq 2} n(n-1) t^{n-2}+\sum_{n \geq 2} n t^{n-2}=v^{\prime \prime}(t)+\sum_{n \geq 0} n t^{n-2}-t^{-1} \\
& =v^{\prime \prime}(t)+\frac{v^{\prime}(t)}{t}-t^{-1}=\frac{3 t-4-t^{2}}{(t-1)^{3}},
\end{aligned}
$$


where $v(t)=\sum_{n \geq 0} t^{n}, v^{\prime}(t)=\sum_{n \geq 0} n t^{n-1}, v^{\prime \prime}(t)=\sum_{n \geq 0} n(n-1) t^{n-2}$. Substituting the above equalities into (3.4), $u(t)$ satisfies the following second order linear differential equation

$$
\left(t^{2}-t^{4}\right) u^{\prime \prime}(t)+\left(7 t-2 t^{2}-9 t^{3}\right) u^{\prime}(t)+\left(8-7 t-15 t^{2}\right) u(t)=\frac{3 t-4-t^{2}}{(t-1)^{3}}
$$

with initial conditions $u(0)=a_{3}=\gamma_{\text {avg }}\left(D_{3}\right)=\frac{1}{2}, u^{\prime}(0)=a_{4}=\gamma_{\text {avg }}\left(D_{4}\right)=\frac{5}{6}$.

With the help of a computer algebra systems, the solution of the above equation is

$$
u(t)=\frac{1}{4(t-1) t^{2}}+\frac{w(t)}{4(t-1)^{2} t^{4}},
$$

where

$$
\begin{aligned}
w(t)=-t^{3}+2 t^{3} \ln (t & +1)+3 t^{2}-2 t^{2} \ln (t+1) \\
& -2 t \ln (1-t)-2 t \ln (t+1)+2 \ln (1-t)+2 \ln (t+1) .
\end{aligned}
$$

By Taylor's formula, we get

$$
\begin{aligned}
\frac{1}{4(t-1) t^{2}}= & \sum_{m \geq-2}\left(-\frac{1}{4}\right) t^{m} \\
w(t)= & t^{2}-t^{3} \\
& +\sum_{m \geq 4} \frac{2\left(4(-1)^{m} m^{2}+m^{2}-12(-1)^{m} m-5 m+6(-1)^{m}+6\right)}{(m-3)(m-2)(m-1) m} t^{m} \\
\frac{1}{4(t-1)^{2} t^{4}}= & \sum_{m \geq-4} \frac{m+5}{4} t^{m} .
\end{aligned}
$$

Therefore, comparing the coefficients of $t^{n-3}$ of the both sides of (3.5) gives

$$
\begin{aligned}
a_{n}= & -\frac{1}{4}+\frac{n}{4}-\frac{n-1}{4} \\
& +\sum_{m=4}^{n+1} \frac{2\left(4(-1)^{m} m^{2}+m^{2}-12(-1)^{m} m-5 m+6(-1)^{m}+6\right)}{(m-3)(m-2)(m-1) m} \cdot \frac{n-m+2}{4} \\
= & \frac{n}{2} \sum_{m=4}^{n+1}\left[\frac{(-1)^{m}\left(4 m^{2}-12 m+6\right)}{(m-3)(m-2)(m-1) m}+\frac{\left(m^{2}-5 m+6\right)}{(m-3)(m-2)(m-1) m}\right] \\
& \quad-\sum_{m=4}^{n+1} \frac{(-1)^{m}\left(4 m^{2}-12 m+6\right)+\left(m^{2}-3 m\right)+(-2 m+6)}{(m-3)(m-2)(m-1) m} \cdot \frac{m-2}{2} \\
& \quad \sum_{m=4}^{n+1} \frac{(-1)^{m}\left(4 m^{2}-12 m+6\right)}{(m-3)(m-2)(m-1) m}+\frac{n}{2} \sum_{m=4}^{n+1} \frac{1}{(m-1) m} \\
& -\frac{1}{2} \sum_{m=4}^{n+1} \frac{(-1)^{m}\left(4 m^{2}-12 m+6\right)}{(m-3)(m-1) m}-\frac{1}{2} \sum_{m=4}^{n+1} \frac{1}{m-1}+\sum_{m=4}^{n+1} \frac{1}{m(m-1)}
\end{aligned}
$$




$$
\begin{aligned}
& =\frac{n}{2} \sum_{m=4}^{n+1} \frac{(-1)^{m}\left(4 m^{2}-12 m+6\right)}{(m-3)(m-2)(m-1) m}+\frac{n}{2}\left(\frac{1}{3}-\frac{1}{n+1}\right) \\
& \quad-\frac{1}{2} \sum_{m=4}^{n+1} \frac{(-1)^{m}\left(4 m^{2}-12 m+6\right)}{(m-3)(m-1) m}-\frac{1}{2} \sum_{m=1}^{n+1} \frac{1}{m} \\
& \quad+\frac{3}{4}+\frac{1}{2(n+1)}+\left(\frac{1}{3}-\frac{1}{n+1}\right)
\end{aligned}
$$

which yields the desired result (3.2).

Now we are in a position to prove (3.3). Using the software Mathematica or series theory, one has

$$
\sum_{m=4}^{n+1} \frac{(-1)^{m}\left(4 m^{2}-12 m+6\right)}{(m-3)(m-2)(m-1) m}=\frac{2}{3}+o\left(\frac{1}{n}\right)
$$

and

$$
\sum_{m=4}^{n+1} \frac{(-1)^{m}\left(2 m^{2}-6 m+3\right)}{(m-3)(m-1) m}=\frac{7}{12}+o(1) .
$$

Combining (3.6) - (3.7), (2.10) and (3.2), we complete the proof of (3.3).

\section{Some remarks}

Bouquets and dipoles are two important classes of graphs in topological graph theory. Their average genera are of independent interest. In this paper, we obtain explicit formulas for $\gamma_{\text {avg }}\left(B_{n}\right)$ and $\gamma_{\text {avg }}\left(D_{n}\right)$. By Theorems 2.2 and 3.2, we have the following relation between $\gamma_{\text {avg }}\left(B_{n}\right)$ and $\gamma_{\text {avg }}\left(D_{n}\right)$,

$$
\gamma_{\mathrm{avg}}\left(B_{n}\right)=\gamma_{\mathrm{avg}}\left(D_{n}\right)+\frac{1-\ln 2}{2}+o(1)
$$

It follows that the difference of $\gamma_{\mathrm{avg}}\left(B_{n}\right)$ and $\gamma_{\mathrm{avg}}\left(D_{n}\right)$ tends to the constant $\frac{1-\ln 2}{2}$ when $n$ tends to infinity.

Since both $B_{n}$ and $D_{n}$ are upper-embeddable, the maximum genera of $B_{n}$ and $D_{n}$ are $\left\lfloor\frac{n}{2}\right\rfloor$ and $\left\lfloor\frac{n-1}{2}\right\rfloor$, respectively. Recall that the minimum genera of $B_{n}$ and $D_{n}$ equal 0 . Therefore, also by Theorems 2.2 and 3.2, we have

$$
\lim _{n \rightarrow \infty} \frac{\gamma_{\text {avg }}\left(B_{n}\right)}{\left\lfloor\frac{n}{2}\right\rfloor}=1 \text { and } \lim _{n \rightarrow \infty} \frac{\gamma_{\text {avg }}\left(D_{n}\right)}{\left\lfloor\frac{n-1}{2}\right\rfloor}=1 .
$$

This implies that the average genus of $B_{n}\left(D_{n}\right)$ is closer to the maximum genus than to the minimum genus.

\section{ORCID iDs}

Xuhui Peng (D) https://orcid.org/0000-0002-2443-4896

\section{References}

[1] J. Chen, A linear-time algorithm for isomorphism of graphs of bounded average genus, SIAM J. Discrete Math. 7 (1994), 614-631, doi:10.1137/s0895480191196769. 
[2] J. Chen and J. L. Gross, Limit points for average genus. I. 3-connected and 2-connected simplicial graphs, J. Comb. Theory Ser. B 55 (1992), 83-103, doi:10.1016/0095-8956(92)90033-t.

[3] J. Chen and J. L. Gross, Kuratowski-type theorems for average genus, J. Comb. Theory Ser. B 57 (1993), 100-121, doi:10.1006/jctb.1993.1009.

[4] J. Chen, J. L. Gross and R. G. Rieper, Lower bounds for the average genus, J. Graph Theory 19 (1995), 281-296, doi:10.1002/jgt.3190190302.

[5] Y. Chen, Lower bounds for the average genus of a CF-graph, Electron. J. Combin. 17 (2010), \#R150 (14 pages), doi:10.37236/422.

[6] L. Comtet, Advanced Combinatorics, Springer Netherlands, 1974, doi:10.1007/ 978-94-010-2196-8.

[7] R. L. Graham, D. E. Knuth and O. Patashnik, Concrete Mathematics: A Foundation for Computer Science, Addison-Wesley, Reading, MA, 1989.

[8] J. L. Gross, Every connected regular graph of even degree is a Schreier coset graph, J. Comb. Theory Ser. B 22 (1977), 227-232, doi:10.1016/0095-8956(77)90068-5.

[9] J. L. Gross and M. L. Furst, Hierarchy for imbedding-distribution invariants of a graph, $J$. Graph Theory 11 (1987), 205-220, doi:10.1002/jgt.3190110211.

[10] J. L. Gross, E. W. Klein and R. G. Rieper, On the average genus of a graph, Graphs Combin. 9 (1993), 153-162, doi:10.1007/bf02988301.

[11] J. L. Gross, D. P. Robbins and T. W. Tucker, Genus distributions for bouquets of circles, J. Comb. Theory Ser. B 47 (1989), 292-306, doi:10.1016/0095-8956(89)90030-0.

[12] J. L. Gross and T. W. Tucker, Generating all graph coverings by permutation voltage assignments, Discrete Math. 18 (1977), 273-283, doi:10.1016/0012-365x(77)90131-5.

[13] J. L. Gross and T. W. Tucker, Topological Graph Theory, Wiley-Interscience Series in Discrete Mathematics and Optimization, John Wiley \& Sons, New York, 1987.

[14] J. H. Kwak and S. H. Shim, Total embedding distributions for bouquets of circles, Discrete Math. 248 (2002), 93-108, doi:10.1016/s0012-365x(01)00187-x.

[15] K. McGown and A. Tucker, Statistics of genus numbers of cubic fields, 2016, arXiv:611.07088 [math.NT].

[16] R. Riper, The enumeration of graph embeddings, Ph.D. thesis, Western Michigan University, 1990, https: / / scholarworks.wmich.edu/dissertations/2105.

[17] S. Stahl, The average genus of classes of graph embeddings, Congr. Numer. 40 (1983), 375388, proceedings of the Fourteenth Southeastern Conference on Combinatorics, Graph Theory and Computing (Boca Raton, Florida, 1983).

[18] S. Stahl, Region distributions of graph embeddings and Stirling numbers, Discrete Math. 82 (1990), 57-78, doi:10.1016/0012-365x(90)90045-j.

[19] S. Stahl, Permutation-partition pairs. III. Embedding distributions of linear families of graphs, J. Comb. Theory Ser. B 52 (1991), 191-218, doi:10.1016/0095-8956(91)90062-o.

[20] S. Stahl, Bounds for the average genus of the vertex-amalgamation of graphs, Discrete Math. 142 (1995), 235-245, doi:10.1016/0012-365x(93)e0221-o.

[21] A. T. White, Graphs, Groups and Surfaces, volume 8 of North-Holland Mathematics Studies, North-Holland Publishing Company, Amsterdam, 2nd edition, 1984.

[22] A. T. White, An introduction to random topological graph theory, Combin. Probab. Comput. 3 (1994), 545-555, doi:10.1017/s0963548300001395. 